\title{
The pill toolbox: How to choose a combined oral contraceptive
}

\author{
A thorough understanding of the risks and benefits, including \\ noncontraceptive advantages, of varied COC formulations strengthens \\ your pill armamentarium and aids patient decision making
}

\section{Charlotte M. Page, MD}

\section{IN THIS ARTICLE}

\section{Common patient concerns}

this page

\section{Recommended cOCs for varied patient problems page 29}

Minimizing AEs page 30 $\mathrm{n}$ the era of long-acting reversible contraceptives (LARCs), the pill can seem obsolete. However, it is still the second most commonly used birth control method in the United States, chosen by $19 \%$ of female contraceptive users as of 2015-2017. ${ }^{1}$ It also has noncontraceptive benefits, so it is important that obstetrician-gynecologists are wellversed in its uses. In this article, I will focus on combined oral contraceptives (COCs; TABLE 1, page 27), reviewing the major risks, benefits, and adverse effects of COCs before focusing on recommendations for particular formulations of COCs for various patient populations.

\section{Benefits and risks}

There are numerous noncontraceptive benefits of COCs, including menstrual cycle regulation; reduced risk of ovarian, endometrial, and colorectal cancer; and treatment of menorrhagia, dysmenorrhea, acne, menstrual migraine, premenstrual syndrome and premenstrual dysphoric disorder, pelvic pain due to endometriosis, and hirsutism.

Dr. Page is Instructor in the Department of Obstetrics, Gynecology and Reproductive Biology, Brigham and Women's Hospital, Boston, Massachusetts.

The author reports no financial relationships relevant to this article.

doi: 10.12788/obgm.0056

\section{Common patient concerns}

In terms of adverse effects, there are more potential unwanted effects of concern to women than there are ones validated in the literature. Accepted adverse effects include nausea, breast tenderness, and decreased libido. However, one of the most common concerns voiced during contraceptive counseling is that COCs will cause weight gain. A 2014 Cochrane review identified 49 trials studying the weight gain question. ${ }^{2}$ Of those, only 4 had a placebo or nonintervention group. Of these 4 , there was no significant difference in weight change between the COC-receiving group and the control group. When patients bring up their concerns, it may help to remind them that women tend to gain weight over time whether or not they are taking a COC.

Another common concern is that COCs cause mood changes. A 2016 review by Schaffir and colleagues sheds some light on this topic, ${ }^{3}$ albeit limited by the paucity of prospective studies. This review identified only 1 randomized controlled trial comparing depression incidence among women initiating a $\mathrm{COC}$ versus a placebo. There was no difference in the incidence of depression among the groups at 3 months. Among 4 large retrospective studies of women using COCs, the agents either had no or a beneficial effect on mood. Schaffir's review reports that there may be greater mood adverse effects with COCs among women with underlying mood disorders. 
TABLE 1 Common brand names of combined oral contraceptives and their components

\begin{tabular}{|c|c|c|c|}
\hline Brand name & Progestin (mg) & Estrogen $(\mu \mathrm{g})$ & Cycle \\
\hline Yaz & Drospirenone (3) & Ethinyl estradiol (20) & $24 / 4$ \\
\hline Yasmin & Drospirenone (3) & Ethinyl estradiol (30) & $21 / 7$ \\
\hline Amethyst & Levonorgestrel (0.09) & Ethinyl estradiol (20) & $\begin{array}{l}\text { No placebo pills; can be used } \\
\text { continuously }\end{array}$ \\
\hline $\begin{array}{l}\text { Loestrin Fe 1/20 } \\
\text { Junel Fe 1/20 }\end{array}$ & Norethindrone (1) & Ethinyl estradiol (20) & $\begin{array}{l}21 / 7 \text { ferrous fumarate instead } \\
\text { of placebo }\end{array}$ \\
\hline $\begin{array}{l}\text { Loestrin 1.5/30 } \\
\text { Junel 1.5/30 }\end{array}$ & Norethindrone (1.5) & Ethinyl estradiol (30) & $\begin{array}{l}21 / 7 \text {. Also available with } \\
\text { ferrous fumarate. }\end{array}$ \\
\hline $\begin{array}{l}\text { Sprintec } \\
\text { Ortho-Cyclen }\end{array}$ & Norgestimate $(0.25)$ & Ethinyl estradiol (35) & $21 / 7$ \\
\hline Lo Loestrin Fe & Norethindrone $(1,0)$ & Ethinyl estradiol $(10,10)$ & $\begin{array}{l}\text { 24/2/2 ferrous fumarate } \\
\text { (biphasic) }\end{array}$ \\
\hline $\begin{array}{l}\text { Ortho Tri-Cyclen } \\
\text { Tri-Sprintec }\end{array}$ & $\begin{array}{l}\text { Norgestimate }(0.18,0.215 \text {, } \\
0.25)\end{array}$ & Ethinyl estradiol $(35,35,35)$ & 7/7/7/7 (triphasic) \\
\hline Seasonique & Levonorgestrel $(0.15,0)$ & Ethinyl estradiol $(30,10)$ & $84 / 7$ (no true placebo pill) \\
\hline LoSeasonique & Levonorgestrel $(0.1,0)$ & Ethinyl estradiol $(20,10)$ & $84 / 7$ (no true placebo pill) \\
\hline Introvale & Levonorgestrel (0.15) & Ethinyl estradiol (30) & $84 / 7$ \\
\hline
\end{tabular}

Patients may worry that COC use will permanently impair their fertility or delay return to fertility after discontinuation. Research does indicate that return of fertility after stopping COCs often takes several months (compared with immediate fertility after discontinuing a barrier method). However, there still seem to be comparable conception rates within 12 months after discontinuing COCs as there are after discontinuing other common nonhormonal or hormonal contraceptive methods. Fertility is not impacted by the duration of COC use. In addition, return to fertility seems to be comparable after discontinuation of extended cycle or continuous COCs compared with traditional-cycle COCs. ${ }^{4}$

\section{COC safety}

Known major risks of COCs include venous thromboembolism (VTE). The risk of VTE is about double among COC users than among nonpregnant nonusers: $3-9$ per 10,000 woman-years compared with $1-5 .{ }^{5}$ In a study by the US Food and Drug Administration, drospirenone-containing COCs had double the risk of VTE than other COCs. However, the position of the American College of Obstetricians and Gynecologists on this increased risk of VTE with drospirenone-containing pills is that it is "possible" and "minimal." It is important to remember that an alternative to COC use is pregnancy, in which the VTE risk is about double that among COC users, at 5-20 per 10,000 woman-years. This risk increases further in the postpartum period, to $40-65$ per 10,000 woman-years. ${ }^{5}$

Another known major risk of COCs is arterial embolic disease, including cerebrovascular accidents and myocardial infarctions. Women at increased risk for these complications include those with hypertension, diabetes, and/or obesity and women who are aged 35 or older and smoke. Interestingly, women with migraines with aura are at increased risk for stroke but not for myocardial infarction. These women increase their risk of stroke 2- to 4 -fold if they use COCs. 
FAST

TRACK

For women

with menstrual

migraines

without aura,

any continuous

formulation $\mathrm{COC}$

or the extended

formulations

LoSeasonique

or Lo Loestrin

can help prevent

migraine

\section{Different pills for different problems}

With so many pills on the market, it is important for clinicians to know how to choose a particular pill for a particular patient. The following discussion assumes that the patient in question desires a COC for contraception, then offers guidance on how to choose a pill with patient-specific noncontraceptive benefits (TABLE 2).

When HMB is a concern. Patients with heavy menstrual bleeding may experience fewer bleeding and/or spotting days with extended cyclic or continuous use of a COC rather than with traditional cyclic use ${ }^{6}$ Examples of such COC options include:

- Introvale and Seasonique, both extendedcycle formulations

- Amethyst, which is formulated without placebo pills so that it can be used continuously

- any other COC prescribed with instructions for the patient to skip placebo pills.

An extrapolated benefit to extended-cycle or continuous COCs use for heavy menstrual bleeding is addressing anemia.

For premenstrual dysphoric disorder, the only randomized controlled trials showing improvement involve drospirenone-ethinyl estradiol pills (Yaz and Yasmin). ${ }^{7}$ There is also evidence that extended cyclic or continuous use of these formulations is more impactful for premenstrual dysphoric disorder than a traditional cycle. ${ }^{8}$

Keeping migraine avoidance and prevention in mind. Various studies have looked at the impact of different COC formulations on menstrual-related symptoms. There is evidence of greater improvement in headache, bloating, and dysmenorrhea with extended cyclic or continuous use compared with traditional cyclic use. ${ }^{6}$

In terms of headache, let us delve into menstrual migraine in particular. Menstrual migraines occur sometime between 2 days prior to 2 days after the first day of menses and are linked to a sharp drop in estrogen levels. COCs are contraindicated in women with menstrual migraines with aura because of the increased stroke risk. For women with menstrual migraines without aura, COCs can prevent migraines. Prevention depends on minimizing fluctuations in estrogen levels; any change in estrogen level greater than $10 \mu \mathrm{g}$ of ethinyl estradiol may trigger an estrogen-related migraine. All currently available regimens of COCs that comprise 21 days of active pills and 7 days of placebo involve a drop of more than $10 \mu \mathrm{g}$. Options that involve a drop of $10 \mu \mathrm{g}$ or less include any continuous formulation, the extended formulation LoSeasonique (levonorgestrel $0.1 \mathrm{mg}$ and ethinyl estradiol $20 \mu \mathrm{g}$ for 84 days, then ethinyl estradiol $10 \mu \mathrm{g}$ for 7 days), and Lo Loestrin (ethinyl estradiol $10 \mu \mathrm{g}$ and norethindrone $1 \mathrm{mg}$ for 24 days, then ethinyl estradiol $10 \mu \mathrm{g}$ for 2 days, then placebo for 2 days). ${ }^{9}$

What's best for acne-prone patients? All COCs should improve acne by increasing levels of sex hormone binding globulin. However, some comparative studies have shown drospirenone-containing COCs to be the most effective for acne. This finding makes sense in light of studies demonstrating antiandrogenic effects of drospirenone. ${ }^{10}$

Managing PCOS symptoms. It seems logical, by extension, that drospirenone-containing COCs would be particularly beneficial for treating hirsutism associated with polycystic ovary syndrome (PCOS). Other low-androgenicpotential progestins, such as a third-generation progestin (norgestimate or desogestrel), might similarly be hypothesized to be advantageous. However, there is currently insufficient evidence to recommend any one COC formulation over another for the indication of PCOS.11

Ovarian cysts: Can COCs be helpful? COCs are commonly prescribed by gynecologists for patients with functional ovarian cysts. It is important to note that COCs have not been found to hasten the resolution of existing cysts, so they should not be used for this purpose. ${ }^{12}$ Studies of early COCs, which had high doses of estrogen (on the order of $50 \mu \mathrm{g}$ ), showed lower rates of cysts among users. This effect seems to be attenuated with the lower-estrogen-dose pills that are currently available, but there still appears to be benefit. Therefore, for a patient prone to cysts who desires an oral contraceptive, a COC containing estrogen $35 \mu \mathrm{g}$ is likely to be the most beneficial of COCs currently on the market. ${ }^{13,14}$ 
TABLE 2 Recommended combined oral contraceptives

\section{for different patient problems}

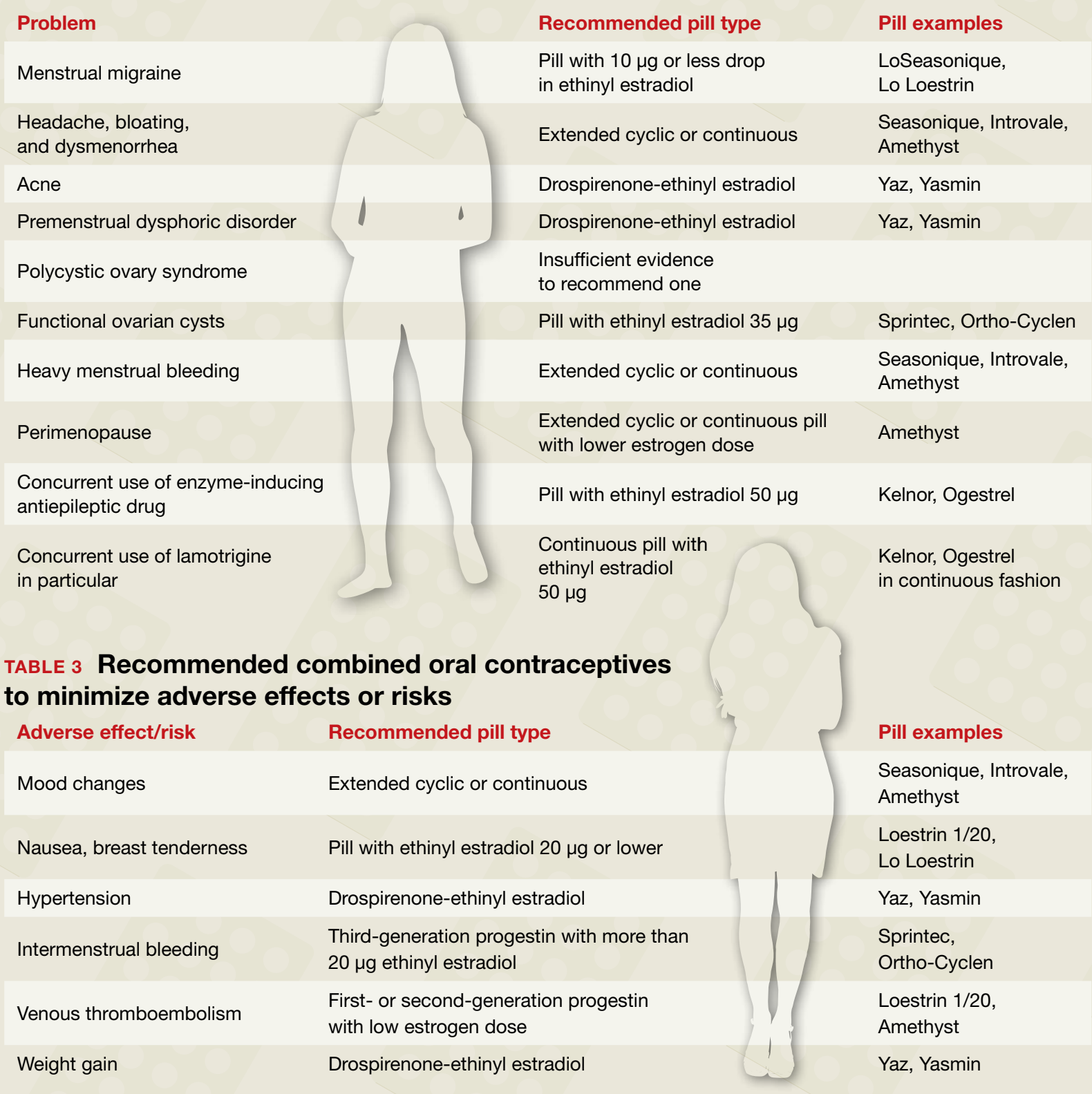

\section{Lower-dosage COCs in perimenopause} may be beneficial. COCs can ameliorate perimenopausal symptoms including abnormal uterine bleeding and vasomotor symptoms. Clinicians are often hesitant to prescribe COCs for perimenopausal women because of increased risk of VTE, stroke, myocardial infarction, and breast cancer with increasing age. However, age alone is not a contraindication to any contraceptive method. An extended cyclic or continuous regimen COC may be the best choice for a perimenopausal woman in order to avoid vasomotor symptoms that occur during hormone-free intervals. In addition, given the increasing risk of adverse effects like VTE with estrogen dose, a lower estrogen formulation is advisable. ${ }^{15}$

Patients with epilepsy who are taking antiepileptic drugs (AEDs) are a special population when it comes to COCs. Certain AEDs induce hepatic enzymes involved in the metabolism and protein binding of 
FAST

TRACK

For obese women
who are high
risk for VTE
but medically
appropriate for
a COC, the best
options are a
first- or second-
generation
progestin
combined with
a low-dose
estrogen

For obese women who are high

risk for VTE

but medically

appropriate for

a COC, the best

options are a

first- or second-

generation

progestin

combined with

estrogen
COCs, which can result in contraceptive failure. Strong inducers are carbamazepine, oxcarbazepine, perampanel, phenobarbital, phenytoin, and primidone. Weak inducers are clobazam, eslicarbazepine, felbamate, lamotrigine, rufinamide, and topiramate. Women taking any of the above AEDs are recommended to choose a different form of contraception than a COC. However, if they are limited to COCs for some reason, a preparation containing estrogen $50 \mu \mathrm{g}$ is recommended. It is speculated that the efficacy and adverse effects of COCs with increased hormone doses, used in combination with enzyme-inducing AEDs, should be comparable to those with standard doses when not combined with AEDs; however, this speculation is unproven. ${ }^{16}$ There are few COCs on the market with estrogen doses of $50 \mu \mathrm{g}$, but a couple of examples are Kelnor and Ogestrel.

Additional factors have to be considered with concurrent COC use with the AED lamotrigine since COCs increase clearance of this agent. Therefore, patients taking lamotrigine who start COCs will need an increase in lamotrigine dose. To avoid fluctuations in lamotrigine serum levels, use of a continuous COC is recommended. ${ }^{17}$

\section{Pill types to minimize adverse effects or risks}

For women who desire to use a COC for contraception but who are at risk for a particular complication or are bothered by a particular adverse effect, ObGyns can optimize the choice of pill (TABLE 3, page 29). For example, women who have adverse effects of nausea and/or breast tenderness may benefit from reducing the estrogen dose to $20 \mu \mathrm{g}$ or lower. ${ }^{18}$

\section{Considering VTE}

As discussed previously, VTE is a risk with all COCs, but some pills confer greater risk than others. For one, VTE risk increases with estrogen dose. In addition, VTE risk depends on the type of progestin. Drospirenone and third-generation progestins (norgestimate, gestodene, and desogestrel) confer a higher risk of VTE than first- or second-generation progestins. For example, a pill with estradiol $30 \mu \mathrm{g}$ and either a third-generation progestin or drospirenone has a $50 \%$ to $80 \%$ higher risk of VTE compared with a pill with estradiol $30 \mu \mathrm{g}$ and levonorgestrel.

For patients at particularly high risk for VTE, COCs are contraindicated. For patients for whom COCs are considered medically appropriate but who are at higher risk (eg, obese women), it is wise to use a pill containing a first-generation (norethindrone) or second-generation progestin (levonorgestrel) combined with the lowest dose of estrogen that has tolerable adverse effects. ${ }^{19}$

\section{What about hypertension concerns?}

Let us turn our attention briefly to hypertension and its relation to COC use. While the American College of Cardiology and the American Heart Association redefined hypertension in 2017 using a threshold of 130/80 $\mathrm{mm} \mathrm{Hg}$, the American College of Obstetricians and Gynecologists (ACOG) considers hypertension to be 140/90 mm Hg in terms of safety of using COCs. ACOG states, "women with blood pressure below 140/90 mm Hg may use any hormonal contraceptive method." ${ }^{20}$ In women with hypertension in the range of 140-159 $\mathrm{mm} \mathrm{Hg}$ systolic or 90-99 mm Hg diastolic, COCs are category 3 according to the US Medical Eligibility Criteria for Contraceptive Use, meaning that the risks usually outweigh the benefits. For women with blood pressures of 160/110 mm Hg or greater, COCs are category 4 (contraindicated). If a woman with mild hypertension is started on a COC, a drospirenone-containing pill may be the best choice because of its diuretic effects. While other contemporary COCs have been associated with a mild increase in blood pressure, drospirenone-containing pills have not shown this association. ${ }^{21}$

\section{At issue: Break-through bleeding, mood, and weight gain}

For women bothered by intermenstrual bleeding, use of a COC with a third-generation progestin may be preferable to use of one with a first- or second-generation. It may be because of decreased abnormal bleeding that COCs with third-generation progestins have lower 
discontinuation rates. ${ }^{22}$ In addition, COCs containing estrogen $20 \mu \mathrm{g}$ or less are associated with more intermenstrual bleeding than those with more than $20 \mu \mathrm{g}$ estrogen..$^{23}$ Keep in mind that it is common with any COC to have intermenstrual bleeding for the first several months.

For women with pre-existing mood disorders or who report mood changes with COCs, it appears that fluctuations in hormone levels are problematic. Consistently, there is evidence that monophasic pills are preferable to multiphasic and that extended cyclic or continuous use is preferable to traditional cyclic use for mitigating mood adverse effects. There is mixed evidence on whether a low dose of ethinyl estradiol is better for mood. ${ }^{3}$

Although it is discussed above that randomized controlled trials have not shown an association between COC use and weight gain, many women remain concerned. For these women, a drospirenone-containing COC may be the best choice. Drospirenone has antimineralocorticoid activity, so it may help prevent water retention.

\section{A brief word about multiphasic COCs.} While these pills were designed to mimic physiologic hormone fluctuations and minimize hormonal adverse effects, there is insufficient evidence to compare their effects to those of monophasic pills. ${ }^{24}$ Without such evidence, there is little reason to recommend a multiphasic pill to a patient over the more straightforward monophasic formulation.

\section{Conclusion}

There are more nuances to prescribing an optimal COC for a patient than may initially come to mind. It is useful to remember that any formulation of pill may be prescribed in an extended or continuous fashion, and there are benefits for such use for premenstrual dysphoric disorder, heavy menstrual bleeding, perimenopause, and menstrual symptoms. Although there are numerous brands of COCs available, a small cadre will suffice for almost all purposes. Such a "toolbox" of pills could include a pill formatted for continuous use (Seasonique), a low estrogen pill (Loestrin), a drospirenone-containing pill (Yaz), and a pill containing a third-generation progestin and a higher dose of estrogen (Sprintec).

\section{References}

1. Daniels K, Abma JC. Current contraceptive status among women aged 15-49: United States, 2015-2017. NCHS Data Brief, no 327. Hyattsville, MD; 2018.

2. Gallo MF, Lopez LM, Grimes DA, et al. Combination contraceptives: effects on weight. Cochrane Database Syst Rev. 2014:CD003987.

3. Schaffir J, Worly BL, Gur TL. Combined hormonal contraception and its effects on mood: a critical review. Eur Contracept Reprod Health Care. 2016;21:347-355.

4. Barnhart KT, Schreiber CA. Return to fertility following discontinuation of oral contraceptives. Fertil Steril. 2009;91:659-663.

5. American College of Obstetricians and Gynecologists. Committee Opinion \#540: Risk of Venous Thromboembolism AmongUsers of Drospirenone-Containing Oral Contraceptive Pills. Obstet Gynecol. 2012;120:1239-1242.

6. Edelman A, Micks E, Gallo MF, et al. Continuous or extended cycle vs. cyclic use of combined hormonal contraceptives for contraception. Cochrane Database Syst Rev. 2014:CD004695.

7. American College of Obstetricians and Gynecologists. Practice Bulletin \#110: Noncontraceptive Uses of Hormonal Contraceptives. Obstet Gynecol. 2010:206-218.

8. Coffee AL, Kuehl TJ, Willis S, et al. Oral contraceptives and premenstrual symptoms: comparison of a $21 / 7$ and extended regimen. Am J Obstet Gynecol. 2006;195:1311-1319.

9. Calhoun $\mathrm{AH}$, Batur P. Combined hormonal contraceptives and migraine: an update on the evidence. Cleve Clin J Med. 2017;84:631-638.

10. Arowojolu AO, Gallo MF, Lopez LM, et al. Combined oral contraceptive pills for treatment of acne. Cochrane Database Syst Rev. 2012:CD004425.

11. McCartney CR, Marshall JC. CLINICAL PRACTICE. Polycystic Ovary Syndrome. N Engl J Med. 2016;375:54-64.

12. Grimes DA, Jones LB, Lopez LM, et al. Oral contraceptives for functional ovarian cysts. Cochrane Database Syst Rev. 2014:CD006134.

13. Grimes DA, Godwin AJ, Rubin A, et al. Ovulation and

follicular development associated with three low-dose ora contraceptives: a randomized controlled trial. Obstet Gynecol. 1994;83:29-34.

14. Christensen JT, Boldsen JL, Westergaard JG. Functional ovarian cysts in premenopausal and gynecologically healthy women. Contraception. 2002;66:153-157.

15. Hardman SM, Gebbie AE. Hormonal contraceptive regimens in the perimenopause. Maturitas. 2009;63:204-212.

16. Zupanc ML. Antiepileptic drugs and hormonal contraceptives in adolescent women with epilepsy. Neurology. 2006;66 (6 suppl 3):S37-S45.

17. Wegner I, Edelbroek PM, Bulk S, et al. Lamotrigine kinetics within the menstrual cycle, after menopause, and with oral contraceptives. Neurology. 2009;73:1388-1393.

18. Stewart M, Black K. Choosing a combined oral contraceptive pill. Australian Prescriber. 2015;38:6-11.

19. de Bastos M, Stegeman BH, Rosendaal FR, et al. Combined oral contraceptives: venous thrombosis. Cochrane Database Syst Rev. 2014:CD010813.

20. American College of Obstetricians and Gynecologists. Practice Bulletin \#206: use of hormonal contraception in women with coexisting medical conditions. Obstet Gynecol. 2019;133:e128-e150.

21. de Morais TL, Giribela C, Nisenbaum MG, et al. Effects of a contraceptive containing drospirenone and ethinylestradiol on blood pressure, metabolic profile and neurohumoral axis in hypertensive women at reproductive age. Eur J Obstet Gynecol Reprod Biol. 2014;182:113-117.

22. Lawrie TA, Helmerhorst FM, Maitra NK, et al. Types of progestogens in combined oral contraception: effectiveness and side-effects. Cochrane Database Syst Rev. 2011:CD004861.

23. Gallo MF, Nanda K, Grimes DA, et al. $20 \mu \mathrm{g}$ versus $>20 \mu \mathrm{g}$ estrogen combined oral contraceptives for contraception. Cochrane Database Syst Rev. 2013:CD003989.

24. van Vliet HA, Grimes DA, Lopez LM, et al. Triphasic versus monophasic oral contraceptives for contraception. Cochrane Database Syst Rev. 2006:CD003553. 